\title{
Estado del conocimiento y amenazas de la tortuga sabanera (Podocnemis vogli, Podocnemididae) en Colombia
}

\author{
Knowledge and threat status of savanna side-necked turtle \\ (Podocnemis vogli, Podocnemididae) in Colombia
}

\author{
Estado do conhecimento e ameaças da tartaruga da savana \\ (Podocnemis vogli, Podocnemididae) na Colômbia
}

Martha Lucía Ortiz-Moreno ${ }^{1 *}$; José A. Rodríguez-Pulido2*

1 Bióloga, Msc, PhD; ${ }^{2}$ Biólogo, Msc, (C) PhD

* Departamento de Biología y Química, Facultad de Ciencias Básicas e Ingenierías, Universidad de los Llanos, Villavicencio, Colombia.

Email: mlortiz@unillanos.edu.co

Recibido: 23 de octubre de 2015

Aceptado:24 de junio de 2016

\section{Resumen}

Podocnemis vogli (Podocnemididae) es una especie de tortuga dulceacuícola, que se distribuye en los Llanos Orientales de Colombia y en Venezuela. Esta especie habita cuerpos de agua lenticos de las sabanas y tiene una dieta principalmente herbívora; durante su ciclo de vida las hembras se desplazan a tierra firme para anidar en época seca. En este periodo de su vida, las hembras son capturadas por los pobladores de la región para consumir su carne y huevos, realizando una explotación cuya magnitud se desconoce. Además del impacto directo a las poblaciones, los cambios en el uso del suelo han generado la destrucción del hábitat de $P$. vogli. El objetivo de este trabajo es analizar el estado actual del conocimiento y amenaza de $P$. vogli en Colombia, con el fin de estimular su estudio y el establecimiento de medidas para su preservación. La información disponible en las bases de datos académicas muestra que existe un gran vacío en el conocimiento ecológico y genético de $P$. vogli y que es urgente establecer medidas tendientes a su protección para reducir su probabilidad de extinción.

Palabras clave: Administración ambiental, Conservación de los recursos naturales, Ecología, Protección ambiental, Tortugas.

\begin{abstract}
Podocnemis vogli (Podocnemidae) is a freshwater turtle species, distributed in the Eastern Llanos of Colombia and Venezuela. Inhabits lentic water bodies of the savanna and has a mainly herbivorous diet, during its life cycle, the females move to mainland for nesting in dry season. Currently, females of this species are hunted for their meat and eggs for the local

settlers, in an unknown quantity. Besides the direct impact on populations, changes in land use have led to the destruction of the P. vogli habitat. The aim of this paper is analyze the current state of knowledge and threats of P. vogli in Colombia,
\end{abstract}


in order to encourage the study and the establishment of measures for preservation. The information available in academic presendatabases shows that exist a large gap between the ecological and genetic knowledge of P. vogli, and is imperative establish measures for their protection in order to reduce their probability of extinction.

Key words: Environmental administration, Conservation of natural resources, Ecology, Environmental protection, Turtles.

\begin{abstract}
Resumo
Podocnemis vogli (Podocnemidae) é uma espécie de tartaruga de água-doce, que distribui-se nas savanas orientais da Colômbia e na Venezuela. Habita corpos d'água lênticos e tem uma dieta principalmente herbívora. Durante seu ciclo de vida as fêmeas migram para terra firme onde fazem seus ninhos na época da seca. As fêmeas são caçadas pelos moradores da região por conta de sua carne e ovos, fazendo uma exploração cuja magnitude é desconhecida. Além do impacto direto nas populações, as mudanças no uso da terra têm levado à destruição do habitat de $P$. vogli. O objetivo deste trabalho é analisar o estado atual do conhecimento e ameaça da $P$. vogli na Colômbia, a fim de incentivar o estudo e estabelecimento de medidas para sua preservação. As informações disponíveis nas bases de dados acadêmicas mostram que há uma grande lacuna no conhecimento ecológico e genético de $P$. vogli e é urgente o estabelecimento de medidas para a sua proteção visando reduzir sua probabilidade de extinção.
\end{abstract}

Palavras chave: Administração ambiental, Conservação dos recursos naturais, Ecologia, Proteção ambiental, Tartarugas.

\section{Estado del conocimiento sobre Podocnemis vogli}

Podocnemis vogli (Muller, 1935) es una tortuga Pleurodira de la familia Podocnemididae que se distribuye en la cuenca del Orinoco, en los Llanos Orientales de Colombia y Venezuela, por tanto es considerada con distribución restringida (Staton y Dixon, 1977; Defler y Rodriguez, 1998; Merchán, Fidalgo y Pérez, 1998; Rojas-Runjaic et al., 2007; Barrio-Amorós y Narbaiza, 2008; Cadena, 2011; Gaffney et al., 2011; Cadena et al., 2012; Ceballos-Fonseca, 2012; Páez et al., 2012; Rivas et al., 2012; de la Fuente, Sterli y Maniel, 2013). P. vogli ha sido reportada en las subcuencas del Arauca, Casanare, Guaviare, Meta, Tomo, Bita, Inírida y Vichada (Páez et al., 2012).

Los individuos de $P$. vogli poseen caparazón plano más alto en su parte anterior o media, ovoide con escotadura anterior, espaldar convexo, liso con cinco placas vertebrales, cuatro pares de costales y 12 pares de marginales, también posee una ligera indentación nucal. P. vogli tiene un gran escudo intergular (en el plastrón) completamente separado de los escudos gulares adyacentes; extremidades con cinco dedos, en las anteriores cinco uñas y en las posteriores sólo cuatro. Cabeza de color café, sin manchas claras (amarillas o blancas) sobre la escama interpariental, pero las crías tienen marcas faciales blanquecinas o amarillas pálidas distribuidas a los lados del surco interorbital, sobre el borde posterior y medial del ojo y sobre los márgenes del tímpano. Estas marcas se desvanecen en las hembras adultas y pueden ser retenidas parcialmente por los machos maduros. En la cabeza el surco infraorbital está presente, pero no llega sobre la mandíbula; las escamas suboculares son agrandadas (Avendaño, Muñoz y Varela, 2002; Páez et al., 2012). P. vogli pre- senta dos (ocasionalmente tres) barbillas mentonianas ubicadas a la altura de la sínfisis mandibular, tiene cinco poros axilares y uno inguinal sobre el puente. Además, posee una cola corta con protuberancias córneas (Páez et al., 2012).

Los neonatos son redondeados y de caparazón verde oliva, la cabeza tiene manchas amarillas que desaparecen con el tiempo, el plastrón es amarillo, las patas y cola de color gris verdoso (Ramo, 1982; Páez et al., 2012). Las crías y juveniles poseen una carena medial sobre el segundo y cuarto escudos vertebrales. Tiene los escudos marginales posteriores normales en lugar de levantados y vueltos hacia arriba como en $P$. unifilis y Peltocephalus dumerilianus (Páez et al., 2012).

Filogenéticamente $P$. vogli está relacionada con $P$. sextuberculata y $P$. expansa (Vargas-Ramírez, CastañoMora y Fritz, 2008; Gaffney et al., 2011).

Los individuos de P. vogli habitan los cuerpos de agua lenticos de las sabanas. Su alimentación es principalmente herbívora, aunque también consume peces, crustáceos o heces y carroña, siendo ítems alimenticios fundamentales las gramíneas y cangrejos (Poppiana dentata) (Müller, 1935; Ramo, 1982; Pritchard y Trebbau, 1984; Trepte, 1993).

El dimorfismo sexual es poco aparente en P. vogli, aparte de una mancha amarilla en el hocico de los machos y el mayor tamaño de las hembras, la diferencia más marcada es en la zona precloacal, que en los machos es más larga y gruesa debido a la presencia del pene (Ramo, 1982). El sistema urogenital fue descrito por Hernández-Henao et al., (2013), registrando la presencia de riñones de tipo metanéfrico con numerosos 
glomérulos, que confluyen mediante uréteres a las bolsas cloacales; las dos estructuras están revestidas de un epitelio simple cúbico. Los oviductos presentan estriaciones sin presencia de espermatecas, ni de ensanchamiento a manera de útero. El hemipene presenta 5 secciones y una ranura uretral mediana, la cual conforma un saco espermático (Hernández-Henao, Rodríguez-Pulido y Astwood-Romero, 2013).

La maduración sexual de $P$. vogli, se da en los machos a los 3-4 años y en las hembras a los 9 años. Su temporada de anidación es entre noviembre y febrero, produciendo 3 nidadas por estación con 13 a 15 huevos por nidada. Los nidos tienen forma de bolsa con una pared más cóncava que las otras, la boca mide $6-8 \mathrm{~cm}$ de diámetro y 5.5 a $10 \mathrm{~cm}$ de profundidad (Ramo, 1982; Páez et al., 2012). Los huevos son alargados con cáscaras duras, muy calcificadas y presentan un largo que varía entre 3.7 y $4.8 \mathrm{~cm}$, con un ancho que fluctúa entre 2.1 y $2.9 \mathrm{~cm}$ (Ramo, 1982; Pritchard y Trebbau, 1984; Páez et al., 2012). Los huevos tardan entre 3 meses y 3 meses y 22 días en eclosionar, también algunos neonatos pueden tardar periodos de hasta 11 días en salir del nido (Ramo, 1982; Páez et al., 2012). Las posturas son depredadas principalmente por el mato de agua o lobo pollero (Tupinambis teguixin), caricare (Caracara plancus), zorro (Cerdocyon thous), rabipelado (Didelphis marsupialis) y el hombre. Las hormigas en el caso que los huevos estén rotos se los comen hasta dejarlos huecos (Ramo, 1982).

Los juveniles se desarrollan en cuerpos de agua temporales, luego cuando alcanzan tallas mayores y tienen menor riesgo de ser depredadas, se desplazan a cuerpos de agua permanentes (Ramo, 1982; Merchán, Fidalgo y Pérez, 1998; Lee, 2004).

La obtención de sangre periférica además de los parámetros de química sanguínea de P. vogli ya han sido descritos, al igual que la respuesta de sus linfocitos a agentes mitogénicos. De tal manera, que a partir de la vena femoral se puede obtener hasta $10 \mathrm{ml}$ de sangre de un individuo adulto y se puede inducir la división celular de los linfocitos con fitohemaglutinina (Frair, Mittermeier y Rhodin, 1978; Ortiz y Rodriguez, 2002; Ortiz, Bueno y Rodriguez, 2003). Desde el punto de vista genético, existe un claro conocimiento de las características citogenéticas de la especie, el cariotipo está compuesto por 28 elementos sin cromosomas sexuales, organizado de la siguiente manera: un primer grupo integrado por 4 pares submetacéntricos y 1 subtelocéntrico; un segundo grupo formado por 6 pares metacéntricos y un último grupo compuesto por 3 pares acrocéntricos. Las regiones organizadoras nucleolares se encuentran en el primer par cromosó- mico en una banda intercalar en los brazos cortos. La heterocromatina se concentran en las regiones pericentroméricas y se detectaron polimorfismos (bandas intercalares) en los cromosomas 1,2, 3 y 7 posiblemente asociados a su distribución geográfica (Rhodin, 1978; Ortiz, Rodriguez y Bueno, 2005). Sin embargo, hasta el momento no hay reportes que vinculen las singularidades biogeográficas con el DNA mitocondrial de la especie, con el fin de colaborar en la reubicación de individuos incautados y establecer áreas prioritarias de conservación.

Las tortugas presentan dos sistemas diferentes de determinación sexual, uno de estos es el mecanismo bajo el control del genotipo (GSD) y el otro, mecanismo bajo el control o dependiente de la temperatura (TSD) (Valenzuela y Ceballos, 2012). El sistema de determinación sexual en $P$. vogli no ha sido descrito, pero la ausencia de cromosomas sexuales permite suponer que puede ser determinado por la temperatura, por tanto es necesario establecer los rangos térmicos y su relación con los sexos, esta información es prioritaria si se considera el escenario actual de cambio climático global (Rhodin, 1978; Ortiz, Rodriguez y Bueno, 2005; Páez et al., 2012).

Las poblaciones de $P$. vogli han sido escasamente estudiadas, los principales reportes para densidades poblacionales y ecología en Venezuela fueron los trabajos de Marcellini (1979), Ramo (1982), Merchán, Fidalgo y Pérez (1998). En Colombia, la principal investigación fue la realizada por Alarcón-Pardo (1969). Ramo (1982), registró individuos con una talla de $34 \mathrm{~cm}$ y $4.5 \mathrm{~kg}$ en tanto que (Alarcón-Pardo 1969) reportó que los machos llegan a $22.4 \mathrm{~cm}$ y las hembras a $31 \mathrm{~cm}$ (Paéz et al., 2012). El crecimiento es alométrico disminuye con la edad y es muy irregular, sobre todo en las hembras. Dicha variabilidad puede estar determinada por los factores ambientales. En los machos la tasa de mayor crecimiento tiene lugar en julio y las hembras presentan un valor más o menos uniforme desde abril hasta octubre. Las hembras alcanzan su tamaño máximo a los 20 años y los machos casi a los 17 años (Ramo, 1982).

La limitada información ecológica ha imposibilitado la descripción del rango de hogar y requerimientos ambientales necesarios para la conservación de la especie, sin embargo se ha observado que $P$. vogli es una tortuga diurna con movimientos asociados al cuerpo de agua donde habita, también presenta desplazamiento estacional y se lleva a cabo entre la sabana inundada en época de lluvias y los caños o lagunas en época seca. En cuanto a movimientos individuales se detectó como mayor distancia recorrida $10 \mathrm{~km}$, reali- 
zada por un macho en Venezuela (Ramo, 1982). Esto indicaría que el tamaño mínimo para una unidad de conservación debería ser de 100 km² (Ramo, 1982).

\section{Vulnerabilidad de P. vogli}

Podocnemis vogli actualmente se encuentra ubicada en el apéndice II del CITES y en la categoría NT (casi amenazada) en virtud de su amplia distribución en las sabanas de Colombia y Venezuela. En 2002, en el Libro Rojo de Reptiles de Colombia fue considerada como DD (datos deficientes), luego como especie LC (preocupación menor) en la versión global, como VU (vulnerable) en la revisión para Suramérica de este mismo libro en 2010 y en 2015 P. vogli se considera como especie LC (Páez et al., 2012; Morales-Betancourt, Lasso et al., 2015). Las diferencias en la valoración del grado de vulnerabilidad de $P$. vogli obedecen principalmente a la falta de información y su grado de explotación aún no cuantificado (Castaño-Mora, 1997; Castaño-Mora, 2002; Asociación Colombiana de Herpetologia, 2011; Páez et al., 2012). La especie se encuentra amenazada por la caza durante la estación seca y en la época de anidación, cuando las hembras salen de los cuerpos de agua para anidar en el interior de la sabana (Ojasti, 1993; Lee, 2004). La caza de P. vogli ha sido reportada desde 1890 por el Padre José de Calasanz Vela, quien en su travesía por el rio Ariari (Colombia) observo el consumo de esta tortuga por parte de los indígenas Mitúas (Ministerio del Medio Ambiente, 2002). La cacería de las hembras lleva a alterar la relación de sexos en las poblaciones, en Venezuela se determinó una relación de 2.78 machos por hembra, indicando una remoción del $28 \%$ de las hembras, por parte de los habitantes de la región del Apure (Ramo, 1982).

La cacería de tortugas como fuente de proteína es tradicional para los pobladores de las sabanas de Colombia y Venezuela, tanto indígenas como colonos, y fue estimulada desde 1605 por el Papa Pablo V, al considerarla una forma de "pez" junto con reptiles como la iguana, que puede ser consumida en Semana Santa sin temor de cometer pecado (Rodríguez, 2000). Actualmente la crisis socio-económica a aumentado el consumo de las tortugas, al reducirse el poder adquisitivo en las poblaciones rurales (Rodríguez, 2000; Ministerio del Medio Ambiente, 2002; Ceballos-Fonseca, 2012).

En el Orinoco Medio de Venezuela 4.6\% de las tortugas consumidas corresponden a P. vogli (Rodríguez, 2000), en Colombia no hay reportes de la magnitud de la caza para consumo en comunidades locales. Pero la disminución de las poblaciones de otras especies de Podocnemididos como $P$. expansa y $P$. unifilis ha incen- tivado la caza de $P$. vogli a pesar de su menor tamaño (Páez et al., 2012; De La Ossa, 2015). Adicionalmente los juveniles de P. vogli son capturados con el fin de convertirlos en mascotas, al igual que los de $P$. unifilis, ya que son dóciles y de apariencia atractiva (Lee 2004; Peñaloza et al., 2013).

P. vogli antes abundante en los caños y riachuelos de las sabanas de Colombia, ha sido exterminada en amplios sectores de los departamentos del Meta, Arauca y Casanare, como resultado de la cacería y la destrucción de su hábitat natural, especialmente por el establecimiento de cultivos a escala comercial como el arroz y la palma africana (Ministerio del Medio Ambiente, 2002; Quintero Espino (com pers 15-06-2013) (Corporinoquia), 2013).

\section{Tráfico de $P$. vogli}

La magnitud del impacto de la extracción de $P$. vogli en Colombia es desconocido. Según datos de la Policía Ambiental y Ecológica Nacional, entre 2008 y 2013, se han recuperado 23.663 tortugas en todo el país, de las cuales 22.621 son representantes de la familia Podocnemididae, siendo la familia de tortugas más afectada por el tráfico de especies silvestres.

La Policía Metropolitana de Villavicencio, que tiene jurisdicción en los municipios de Villavicencio, Acacias, Cumaral y Restrepo en el departamento del Meta, ha recuperado 197 tortugas entre 2008 y 2013. Sin embargo, por dificultades técnicas la Policía Metropolitana no posee la identificación de la especie a la que pertenecen los individuos.

La Corporación Ambiental Regional-CAR CORMACARENA, que tiene jurisdicción en el departamento del Meta, registra la recuperación de 109 animales vivos de Podocnemis vogli entre los años 2008 a 2015. Entre 2010 a 2012 la CAR CORPORINOQUIA registro 26 individuos de $P$. vogli incautados en su jurisdicción en los departamentos de Arauca, Vichada y Casanare (Tabla 1). Estos datos indican que en los Llanos Colombianos la magnitud del tráfico de tortugas Podocnemididae puede estar subestimado, ya que como lo indica Lee (2004) y Ceballos-Fonseca (2012) P. vogli es principalmente destinada al consumo local y por ello puede no ser registrada en los decomisos policiales (Lee, 2004; Ceballos-Fonseca, 2012; Páez et al., 2012).

Actualmente la caza comercial de tortugas en Colombia es ilegal, ya que el Ministerio de Ambiente y Desarrollo Sostenible de Colombia no ha establecido las especies de tortugas que pueden ser explotadas y los volúmenes de aprovechamiento, indispensable para 
no poner en riesgo a las especies, tal y como lo establece el numeral 42 del artículo 5 de la ley 99 de 1993 y el decreto 4688 de 2005 que reglamenta el Código Nacional de Recursos Naturales Renovables y de Protección al Medio Ambiente y la Ley 611 de 2000.

Los individuos recuperados mediante incautaciones por las autoridades policiales son entregados a las CAR, quienes las encaminan a centros de valoración sanitaria, rehabilitación y finalmente se destinan a la liberación, tal y como lo establece la normatividad vigente (Bonilla et al., 2012). Cormacarena envía los ejemplares al Parque Zoológico "Los Ocarros" en Villavicencio (Meta) o a fincas registradas. Sin embargo, las CAR no realizan seguimiento de las poblaciones receptoras, en cuanto a la dinámica genética y epidemiológica, debido a la falta de recursos económicos y humanos, por lo tanto el impacto de estas liberaciones es desconocido (Páez et al., 2012).

\section{Conservación de $P$. vogli}

Considerando la vulnerabilidad de las diferentes especies de tortugas en Colombia, el entonces Ministerio del Medio Ambiente formuló en 2002, el "Programa nacional para la conservación de las tortugas marinas y continentales de Colombia", orientado hacia el trabajo interinstitucional y la participación de las comunidades. Las líneas de acción del programa eran: 1) investigación y monitoreo de poblaciones; 2) manejo sostenible; 3) educación ambiental y participación comunitaria; 4) información y divulgación; 5) gestión y fortalecimiento institucional. Sin embargo, esta iniciativa tuvo una escaza trascendencia práctica para la mayoría de las especies de tortugas, debido a que su implementación fue parcial y a cargo de iniciativas individuales (Páez et al., 2012).

Frente a este escenario, en 2011 y con el liderazgo de la Asociación Colombiana de Herpetología se inicia la formulación del "Plan estratégico para la conservación de tortugas continentales de Colombia", que rindió sus primeros frutos en el 2012 con el documento sobre "Estrategias para conservación de tortugas continentales de Colombia". En él se presenta el Plan de acción para la cuenca hidrográfica del Orinoco, donde se menciona que en el "Plan de acción de biodiversidad en la cuenca del río Orinoco-Colombia 20052015" (Correa, Ruíz y Arevalo, 2006), Podocnemis vogli es considerada una especie focal para la región. Pero sólo hasta 2007, la Corporación para el desarroIlo sostenible del Norte y Oriente Amazónico-CDA, realiza una evaluación del estado de las poblaciones de especies del género Podocnemis, entre ellas $P$. vogli en la cuenca baja del río Guaviare (Páez et al., 2012).
En 2008, la Fundación Omacha y la Fundación Horizonte Verde en colaboración con otras instituciones elaboran el "Plan de manejo y conservación de especies amenazadas en la reserva de Biosfera El Tuparro", él cuál incluye a $P$. vogli, con líneas de acción similares a las planteadas por el "Programa nacional para la conservación de las tortugas marinas y continentales de Colombia". Los esfuerzos de conservación derivados de este plan se orientaron principalmente hacia la protección de $P$. expansa y $P$. unifilis. Estas especies han sido de interés para el establecimiento de esfuerzos de conservación en Colombia y Venezuela, debido a su potencial comercial y alta vulnerabilidad (MoralesBetancourt, Lasso y Renjifo, 2009; Páez et al., 2012).

La Asociación Colombiana de Herpetología, CORMACARENA y CORPORINOQUIA no registran proyectos de conservación específicos para Podocnemis vogli, debido principalmente a la falta de información ecológica y genética que limita la valoración de la vulnerabilidad de esta especie. La falta de conocimiento biológico también restringe el autocontrol en la extracción de individuos y el desarrollo del sentido de pertenencia en los habitantes del área de distribución de P. vogli, necesarios para el establecimiento de programas de educación ambiental y participación comunitaria, orientados a aumentar su valoración como parte del capital biológico regional que debe ser protegido. Actualmente es ampliamente extendida la creencia de que P. vogli es muy abundante dentro de las CAR y los pobladores de la Cuenca del Orinoco, aunque no existen censos que lo corroboren.

Esta situación de falta de conocimiento biológico, información dispersa y desconocimiento de las causas factibles de la declinación poblacional, es común para otras tortugas continentales y limita el éxito de los programas de conservación. Los pocos esfuerzos de conservación generalmente son puntuales y con baja relevancia para especies longevas como las tortugas (Páez et al., 2012).

\section{Estrategias de conservación}

La literatura considera algunas estrategias para la conservación de quelonios a continuación se analizan sus implicaciones para $P$. vogli:

\section{Unidades de conservación}

Con el desarrollo de proyectos de agricultura comercial, extracción petrolera y los crecientes desarrollos urbanísticos, el hábitat de P. vogli está amenazado por la eliminación de los cuerpos de agua lenticos, la contaminación con agroquímicos y la introducción 
de especies exóticas, además de la sobre explotación humana. Una alternativa es la creación de áreas de preservación para las sabanas nativas de la cuenca del Orinoco, en las cuáles las poblaciones de $P$. vogli estén protegidas del impacto humano (Buhlmann et al., 2009), sin embargo la falta de información biológica limita la disponibilidad de criterios para la selección de estas áreas.

\section{Recuperación de nidadas}

Por otra parte, en el caso de $P$. vogli la recuperación de nidadas y la liberación de neonatos son medidas dif́ciles de establecer, porque la anidación no es gregaria como se da en las especies de rio como $P$. unifilis, $P$. expansa y $P$. lewyana, lo cual dificulta el control de las nidadas (Asociación Venezolana de Ecología, 1996; Morales-Betancourt, Lasso y Renjifo, 2009; Páez et al., 2012).

\section{Zoocria}

Dentro de las estrategias de conservación usualmente no se considera la zoocria y las cuotas de extracción como alternativas para el manejo sostenible de las especies amenazadas de tortugas. (Mittermeier (1978) y Ceballos-Fonseca (2012) proponen las granjas con explotación controlada para garantizar la conservación de las poblaciones y ofrecer animales para mantener el consumo tradicional en los habitantes de la cuenca del Orinoco. Actualmente el único zoocriadero de tortugas continentales reconocido por el Ministerio de Ambiente y Desarrollo Sostenible, es la Estación de Biología Tropical Roberto Franco de la Universidad Nacional de Colombia en Villavicencio (Meta), los individuos obtenidos en esta entidad son orientados a fines científicos (Páez et al., 2012).

La cría de tortugas continentales en Colombia es incipiente, ya sea con fines comerciales, de repoblamiento o científicos y falta seguimiento sobre su eficiencia y adhesión a la normatividad. Adicionalmente, se desconoce el número de zoocriaderos no reglamentados, las especies empleadas y el tipo de explotación establecida (Páez et al., 2012).

La cría artesanal de $P$. vogli debe considerar medidas de bioseguridad para el manejo de los animales y sus residuos, debido a que se ha reportado que esta especie es portadora de nematodos intestinales (Buckley, 1969; Khalil y Gibbons, 1988) y que en las lagunas de cría se favorece el desarrollo de bacterias zoonóticas como Salmonella sp. (Pachón, Pulido y Moreno, 2011).

\section{Recomendaciones}

Finalmente, se quiere instar a los investigadores del área herpetológica a aportar al conocimiento de las características biológicas de $P$. vogli, con el fin de ofrecer elementos para formular estrategias adecuadas que permitan conservar la especie con su profundo valor natural y cultural. Por ello a continuación se listan los aspectos prioritarios para el desarrollo de investigaciones en P. vogli según Páez et al (2012) y que son coincidentes con los análisis realizados en este trabajo:

- Distribución real dentro del territorio nacional

- Estructura de las poblaciones

- Monitorear los efectos del cambio climático en la estructura de las poblaciones

- Historia natural de la especie, específicamente para Colombia

- Evaluar la presión por caza

- Proyectos piloto para la reproducción en cautiverio

\section{Agradecimientos}

Los autores queremos expresar nuestra gratitud al Dr. Carlos Parra del grupo de Bióticos de CORMACARENA, al grupo técnico de la sede "La Primavera" y al Ing. Julian Quintero Espino de CORPORINOQUIA por su colaboración, al igual que al Intendente Jefe Hector Arbey Baquero Castro, Jefe del Grupo de Protección Ambiental y Ecológica de la Policía Metropolitana de Villavicencio y al Área de Protección Ambiental y Ecológica de la Policía Nacional de Colombia.

\section{Referencias}

Asociación Colombiana de Herpetologia. 2011. Plan estratégico de conservación para las tortugas continentales colombianas. Nicolás Aristizabal Litografia-Tipografia, Medellín, Colombia, p. 33.

Alarcón-Pardo H. Contribución al conocimiento de la morfología, ecología, comportamiento and distribución geográfica de Podocnemis vogli. Testudinata._Revista de la Academia Colombiana de Ciencias Exactas Físicas y Naturales. 1969;13:303-329.

Avendaño I, Muñoz A, Varela N. Aproximación al Conocimiento sobre la Reproducción de los Quelonios. Reproducción, 2002;3:42-56.

Barrio-Amorós CL, Narbaiza I. Turtles of the Venezuelan Estado Amazonas. Radiata. 2008;17(1):2-19.

Bonilla M.A., Luque N., Cuervo M.A., Barreto L.C., Zuluaga C., Vásquez E.A. et al. 2012. Tortugas terrestres y de agua dulce de Colombia y manejo de los decomisos. Universidad Nacional 
Tabla 1. Número de ejemplares de $P$. vogli y otras especies recuperados por entidades gubernamentales encargadas del control ambiental en Colombia.

\begin{tabular}{|c|c|c|c|c|c|c|c|c|c|}
\hline Entidad Año & 2008 & 2009 & 2010 & 2011 & 2012 & 2013 & 2014 & 2015 & Total \\
\hline $\begin{array}{l}\text { Individuos de la familia Podocnemididae } \\
\text { incautados en Colombia por la } \\
\text { Policía Ambiental y Ecológica }\end{array}$ & 7325 & 3385 & 5555 & 3309 & 1970 & 1077 & * & * & 22621 \\
\hline $\begin{array}{l}\text { Indivíduos de } P \text {. vogli incautados o } \\
\text { entregados a CORMACARENA }\end{array}$ & 14 & 17 & 19 & 1 & 19 & 10 & 12 & 17 & 109 \\
\hline $\begin{array}{l}\text { Indivíduos de P. vogli incautados o } \\
\text { entregados a CORPORINOQUIA }\end{array}$ & * & * & 3 & 4 & 19 & * & * & * & 26 \\
\hline $\begin{array}{l}\text { Tortugas incautadas por la Policía } \\
\text { Metropolitana de Villavicencio }\end{array}$ & 55 & 32 & 64 & 23 & 13 & 10 & * & * & 197 \\
\hline
\end{tabular}

*Datos no disponibles

de Colombia; Editorial Universidad Nacional de Colombia: Bogotá, Colombia. p.100

Buckley J. On a remarkable oxyurid nematode, Orientatractis leiperi n. sp.,(Atractidae) from South American tortoise, Podocnemis vogli. Journal of Helminthology. 1969;43(3-4):281-286.

Buhlmann KA, Akre T, Iverson JB, Karapatakis D, Mittermeier RA, Georges A, et al. A global analysis of tortoise and freshwater turtle distributions with identification of priority conservation areas.Journal Information. 2009;8(2):116-149.

Cadena EA. Potential earliest record of podocnemidoid turtles from the Early Cretaceous (Valanginian) of Colombia. Journal of Paleontology. 2011;85(5):877-881.

Cadena EA, Ksepka DT, Jaramillo CA, Bloch JI. New pelomedusoid turtles from the late Palaeocene Cerrejón Formation of Colombia and their implications for phylogeny and body size evolution. Journal of Systematic Palaeontology. 2012; 10(2):313-331.

Castaño-Mora OV. 1997. Status of the tortoises and freshwater turtles of Colombia. Conservation, restoration and management of tortoises and turtles-an international conference. New York Turtle and Tortoise Society. New York, USA. p. 302-306.

Castaño-Mora OV. 2002. Libro rojo de reptiles de Colombia. Instituto Alexander von Humboldt, Universidad Nacional de Colombia. Bogotá, Colombia. p. 160.

Ceballos-Fonseca CP. Tortugas (Testudinata) marinas y continentales de Colombia. Biota Colombiana. 2012;1(2):187-194.

Correa HD, Ruíz SL, Arévalo LM. 2006. Plan de acción en biodiversidad en la Cuenca del Orinoco-Colombia 2005-2015-Propuesta técnica. Corporinoquia, Cormacarena, Instituto Alexander von Humboldt, Unitrópico, Fundación Omacha, Fundación Horizonte Verde, Universidad Javeriana, Unillanos, WWF-Colombia, GTZ-Colombia. Bogotá,Colombia. p. 330.

De la Fuente MS, Sterli J, Maniel I. 2013. North Gondwana Pleurodiran Turtles. In: De la Fuente M.S., Sterli J., Maniel I.(Ed.). Origin, Evolution and Biogeographic History of South American
Turtles, Springer International Publishing. New York, USA. cap. 5, p. 95-131.

De La Ossa J. Efecto de sustitución: una expresión del agotamiento poblacional de quelonios en Barcelos, Amazonas, Brasil. Revista de la Asociacion Colombiana de Ciencias Biológicas. 2015;1(22):61-67.

Defler TR, Rodriguez JV. 1998. La fauna de la Orinoquia. En: Fajardo D. (Ed.). Colombia Orinoco. Fondo FEN Colombia. Bogotá, Colombia. p. 135-165.

Frair W, Mittermeier RA, Rhodin AG. Blood biochemistry and relations among Podocnemis turtles (Pleurodira, Pelomedusidae). Comparative Biochemistry and Physiology Part B: Comparative Biochemistry. 1978;61(1):139-143.

Gaffney ES, Meylan PA, Wood RC, Simons E, De Almeida Campos D. Evolution of the side-necked turtles: the family Podocnemididae.Bulletin of the American Museum of Natural History. 2011;350:1-237.

Hernández-Henao WJ, Rodríguez-Pulido JA, Astwood-Romero JA. Anatomía macroscópica y microscópica del sistema urogenital de la tortuga sabanera Podocnemis vogli Muller, 1935.(Testudines Pelomedusidae). Orinoquia. 2013;17(1):120-133.

Khalil LF, Gibbons LM. Two nematodes, Paratractis hystrix (Diesing, 1851) and Buckleyatractis marinkelli ng, n. sp.(Atractidae: Cosmocercoidea) from Podocnemis spp. in Colombia.Systematic parasitology. 1988;12(3):187-198.

Lee DS. Cultural harvest of the Llanos Side-Neck Turtle, Podocnemis vogli, in the Venezuelan Llanos. The Newsletter of Chelonian Conservationists and Biologists. 2004;8:5-8.

Marcellini DL. 1979. Activity patterns and densities of Venezuelan caiman Caiman crocodilus and pond turtles Podocnemis vogli. Vertebrate Ecology in the Neotropics. Smithsonian, Washington, USA. p. 263-271. 
Merchán M, Fidalgo AM, Pérez C. Podocnemis vogli: Biología y distribución del galápago llanero en Venezuela. Reptilia. 1998;4(18):29-31

Ministerio del Medio Ambiente. 2002. Programa Nacional para la conservación de las tortugas marinas y continentales en Colombia. Imprenta Nacional. Bogotá, Colombia. p. 63.

Mittermeier RA. South America's river turtles: saving them by use." Oryx. 1978;14(3):222-230.

Morales-Betancourt M, Lasso C, Páez V, Bock B. 2015. Libro Rojo de Reptiles de Colombia. Instituto de Investigación de Recursos Biológicos Alexander von Humboldt (IAvH), Universidad de Antioquia. Bogotá, Colombia.

Morales-Betancourt MA, Lasso CA, Renjifo JM. Herpetofauna de la estrella fluvial de Inírida (ríos Inírida, Guaviare, Atabapo y Orinoco), Orinoquia colombiana lista preliminar de especies.Biota Colombiana. 2009;10(1 y 2):171-178.

Müller L. Uber eine neue Podocnemis-Art (Podocnemis vogli) aus Venezuela nebst ergänzenden Bemerkungen uber die systematischen Merkmale der ihr nächstverwandten Arten. Zoologischer Anzeiger. 1935;110(5/6): 97-109.

Ojasti J. 1993. Utilización de la fauna silvestre en América Latina: situación y perspectivas para un manejo sostenible, Food \& Agriculture Organization of the United Nations. p.243.

Ortiz ML, Bueno ML, Rodríguez PA. Agentes mitogénicos para cultivos de linfocitos en quelonios. Orinoquia 2003;7(1-2): 47-49.

Ortiz ML, Rodriguez PA. Evaluación de técnicas de obtención de sangre periférica en quelonios. Orinoquia. 2002;6(2):65-74.

Ortiz ML, Rodriguez PA, Bueno ML. Caracterización citogenética de la tortuga sabanera Podocnemis vogli (Reptilia: Testudinata: Podocnemididae). Acta Biológica Colombiana. 2005;10(1):19.

Páez VP, Morales-Betancourt MA, Lasso CA, Castaño-Mora OV, Bock BC. (Eds). 2012. Biologia y conservación de las tortugas continentales de Colombia. Serie recursos hidrobiológicos y pesqueros continentales de Colombia, Instituto Alexander von
Humboldt, Universidad Nacional de Colombia. Editorial Unibiblos. Bogotá, Colombia. p. 512.

Pachón D, Pulido A, Moreno C. Aislamiento y serotipificación de Salmonella sp. en estanques con Crocodylus intermedius y testudines cautivos en Villavicencio-Colombia. Revista MVZ Córdoba. 2011;16(2):2564-2575.

Peñaloza CL, Hernández O, Espín R, Crowder LB, Barreto GR. Harvest of Endangered Sideneck River Turtles (Podocnemis spp.) in the Middle Orinoco, Venezuela. Copeia. 2013;(1):111-120.

Pritchard PCH, Trebbau P. 1984. The turtles of Venezuela, contributions to herpetology. Society for the Study of Amphibians and Reptiles. Oxford, USA. p. 414.

Ramo C. Biologia del galapago (Podocnemis vogli Muller, 1935) en el hato "El Frio", Llanos de Apure (Venezuela). DOÑANA Acta Vertebrata. 1982;9(3):7-159.

Rhodin AGJ. Karyotipic analysis of the Podocnemis turtles. Copeia 1978;4:723-728.

Rivas GA, Molina CR, Ugueto GN, Barros TR, Barrio-Amoros CL, Kok PJR. Reptiles of Venezuela: an updated and commented checklist. Zootaxa. 2012;3211: 1-64.

Rodríguez JP. Impact of the Venezuelan economic crisis on wild populations of animals and plants. Biological Conservation. 2000;96(2):151-159.

Rojas-Runjaic FJ, Infante Rivero EE, Barrio-Amoros CL, Barros Blanco TR. New distributional records of amphibians and reptiles from Estado Zulia in the Maracaibo basin, Venezuela. Herpetological Review. 2007;38(2):235-236.

Staton MA, Dixon JR. The herpetofauna of the central llanos of Venezuela: noteworthy records, a tentative checklist and ecological notes. Journal of Herpetology. 1977;17-24.

Trepte MJ. Juvenile Schienenschildkröten Podocnemis vogli fressen Wasserschweink ot. Salamandra. 1993;29(2):149-152.

Vargas-Ramírez M, Castaño-Mora OV, Fritz U. Molecular phylogeny and divergence times of ancient South American and Malagasy river turtles (Testudines: Pleurodira: Podocnemididae). Organisms Diversity \& Evolution. 2008;8(5):388-398. 